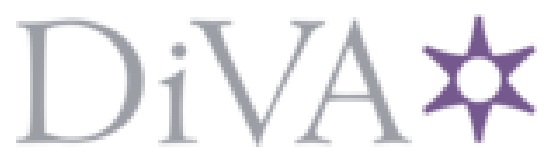

http://www.diva-portal.org

This is the published version of a paper published in International Journal of Sports Medicine.

Citation for the original published paper (version of record):

Díaz, M M., Bocanegra, O L., Teixeira, R R., Soares, S S., Espindola, F S. (2013)

Salivary nitric oxide and alpha-amylase as indexes of training intensity and load International Journal of Sports Medicine, 34(1): 8-13

https://doi.org/10.1055/s-0032-1316318

Access to the published version may require subscription.

N.B. When citing this work, cite the original published paper.

Permanent link to this version:

http://urn.kb.se/resolve?urn=urn:nbn:se:rkh:diva-2792 


\section{Salivary Nitric Oxide and Alpha-Amylase as Indexes of Training Intensity and Load}

Authors

Affiliations

\section{M. Diaz' ${ }^{1}$, O. L. Bocanegra', R. R. Teixeira', S. S. Soares ${ }^{2}$, F. S. Espindola ${ }^{1}$}

${ }^{1}$ Institute of Genetics and Biochemistry, Federal University of Uberlandia, Uberlandia, Brazil

${ }^{2}$ Faculty of Physical Education, Federal University of Uberlandia, Uberlandia, Brazil

\section{Key words \\ - biomarker \\ - saliva \\ - blood \\ - exercise \\ sports training}

accepted after revision

May 10, 2012

\section{Bibliography}

DOI http://dx.doi.org/

10.1055/s-0032-1316318

Published online:

September 7, 2012

Int J Sports Med 2013; 34:

8-13 @ Georg Thieme

Verlag KG Stuttgart · New York ISSN 0172-4622

\section{Correspondence}

Dr. Foued Salmen Espindola

Instituto de Genética e

Bioquímica

Universidade Federal de

Uberlândia

Av. Para 1720

38400982 Uberlândia

Brazil

Tel.: +55/34/3218 2477

Fax: $+55 / 34 / 32182203$

foued@ufu.br

\section{Abstract \\ $\nabla$}

This study examined the variation in salivary nitric oxide (NO), alpha-amylase (SAA) and serum markers of muscle injury during 21 weeks of training in elite swimmers. Samples of saliva and blood were collected once a month during 5 months from 11 male professional athletes during their regular training season. The variation in each marker throughout the 21 weeks was compared with the dynamics of training volume, intensity and load. Unstimulated whole saliva was assessed for NO and SAA whereas venous blood was assessed for lactate dehydrogenase, creatine kinase, and $\gamma$-glutamyltransferase. Nitric

\section{Introduction}

$\nabla$

Sports training is a methodological process that involves systematic oscillations in the volume and intensity of exercise [31]. Periodization allows athletes to reach peaks of performance at predictable times whilst providing a model for controlling recovery and adaptation. Traditionally, monitoring the physiological response to training has been performed using, amongst others, serum markers of muscular damage and variations in hormonal and immune status [16]. The serum levels of lactate dehydrogenase (LDH) and creatine kinase $(\mathrm{CK})$ are widely used in sports medicine to monitor the response and adaptation of skeletal muscle to training [5]. The serum levels of both enzymes generally increase hours after strenuous exercise and return to baseline levels within days of recovery [5]. The concentration of catecholamines is also used as an index of autonomic activity [33]. Overall, during periods of intense training, lower concentrations of catecholamines have been observed in healthy subjects [34]. Although these parameters provide a fairly accurate appraisal of the adaptive oxide and SAA showed a proportional response to the intensity of training. However, whereas the concentration of NO increased across the 21 weeks, the activity of sAA decreased. Similar variations in the concentration of $\mathrm{NO}$ and the markers of muscle injury were also observed. The higher concentration of NO might be attributed to changes in haemodynamics and muscle regenerative processes. On the other hand, autonomic regulation towards parasympathetic predominance might have been responsible for the decrease in sAA activity. These findings provide appealing evidence for the utilization of salivary constituents in sports medicine to monitor training programmes.

response to exercise, the collection of blood specimens is invasive and requires trained personnel. Saliva offers clear advantages over blood in sports research because it is readily available, non-invasive and can be easily collected, handled and stored.

Saliva is produced from 3 major pair of glands and numerous other minor glands spread over the oral mucosa. Most of the components of saliva such as water, electrolytes and proteins, are secreted from the salivary glands under autonomic control. In general, sympathetic nerves are responsible for the secretions of proteins whereas parasympathetic stimulation results predominantly in the secretion of water and electrolytes [26]. Alphaamylase (SAA) is one of the most abundant proteins in saliva and possesses digestive and anti-microbial properties [29]. Because its release into saliva is predominantly regulated by sympathetic control, the response of sAA to exercise has been used as a marker of nervous activity and has been successfully applied to predict exercise intensity in well-trained subjects [3].

Nitric oxide (NO), on the other hand, is synthesized from the amino acid L-arginine by NO syn- 

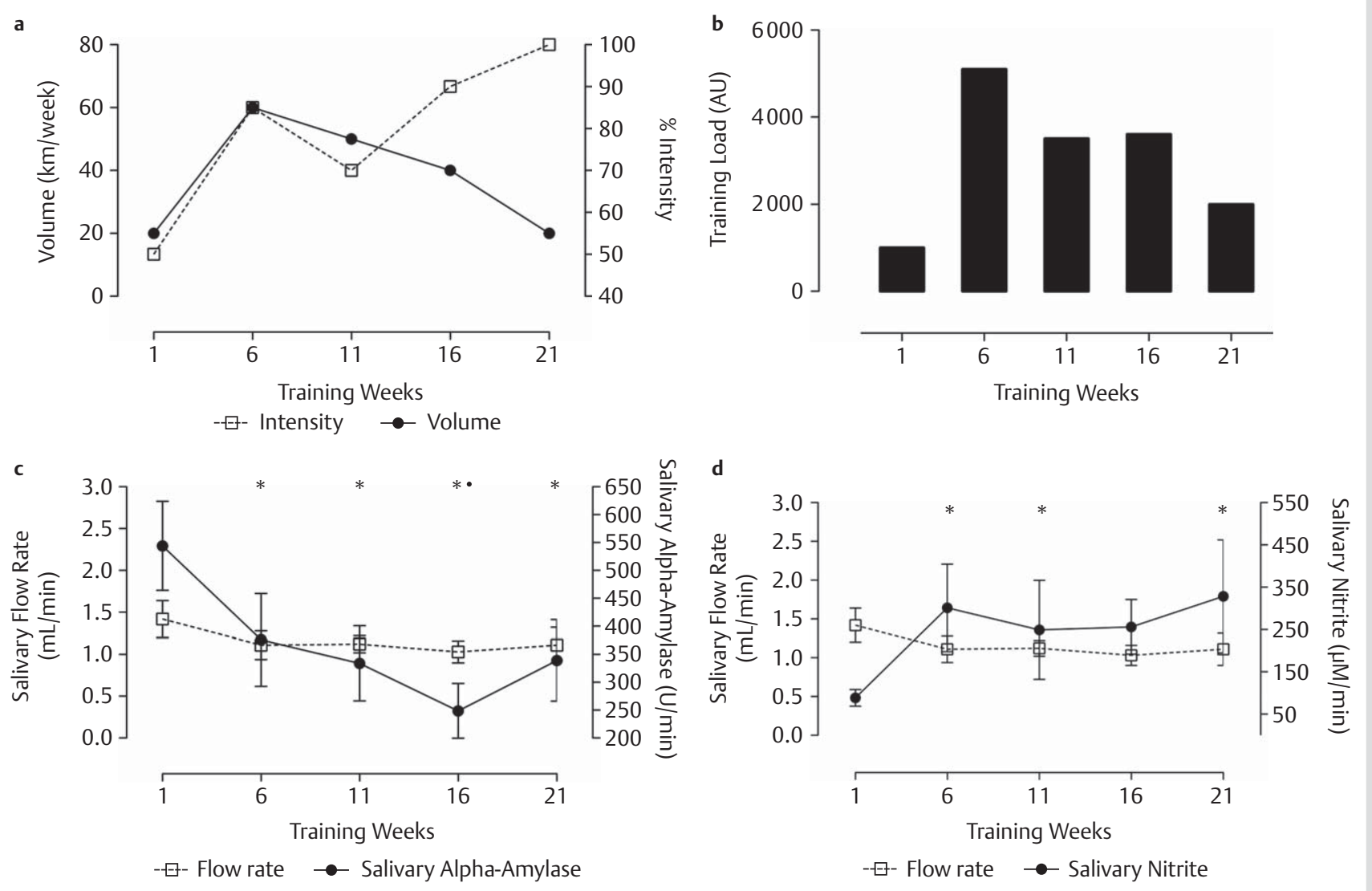

Fig. 1 Variation in training and salivary markers during the 21-week season. Figure a shows the dynamics of volume and intensity of training. Figure $\mathbf{b}$ represents training load (expressed as a function of volume $\times$ intensity). Figures $\mathbf{c}$ and $\mathbf{d}$ show the variation in flow rate and sAA and $\mathrm{NO}$ secretion rate, respectively. *Significantly different from training week 1 at $p<0.05 \bullet$ Significantly different from training week 6 at $p<0.05$.

thase (NOS) and is of major importance in the regulation of coronary and systemic tone [18]. Regular exercise is associated with an enhanced vasodilator capacity and anti-atherogenic effects, which is in part attributed to the up-regulation of NO synthesis [21]. Previous research also has demonstrated that higher levels of NO in plasma during exercise predict exercise capacity in trained subjects [28]. Yet, no study to date has investigated the feasibility of salivary NO to monitor training programmes.

Consequently, although a significant body of research supports the notion that saliva offers an interesting possibility to assess the adaptive response to exercise, missing are studies that enable us to determine the variation of salivary components, mainly NO and sAA, in response to long-term training. This is particularly important in sports medicine since monitoring for abnormal profiles of markers of training status is usually performed in the basal state and not only moments before or after training sessions. Thus, we investigated the variation in salivary $\mathrm{NO}$ and sAA during 21 weeks of training in elite swimmers. We compared their variation with the oscillation of training volume, intensity and load. To gain a better understanding of the impact of training, we also included analysis of heart rate (HR) and the serum markers of muscle damage LDH, CK and $\gamma$-glutamyltransferase $(\gamma-G T)$. Finally, since psychological stimuli might influence the secretion of salivary proteins, mood states across the training season were registered using the Profile of Mood States (POMS) questionnaire. We hypothesized that the variation in SAA and NO would be proportional to the oscillation of the training volume, intensity and load.

\section{Methods}

\section{$\nabla$}

\section{Participants}

The participants were 11 male swimmers (aged 21.5 \pm 2.16 years; BMI: $22.7 \pm 2.5 \mathrm{VO}_{2}$ max: $52.7 \pm 3.2 \mathrm{ml} / \mathrm{kg}$.min; competition experience: $8.7 \pm 2.8$ years) recruited from a professional swimming team before the beginning of a 21-week training season. None of the participants smoked, had significant medical or oral health history or were taking regular or incidental medication during the study. One week before the beginning of the training season, the participants gave their written informed consent. The experimental protocol was in compliance with the ethical standards in sports and exercise science research [13] and was approved by the Institutional Review Board.

\section{Training}

The participants completed 9 training sessions per week that included predominantly swimming. In the swimming sessions, the average distance performed was $9.04 \mathrm{~km}$. Overall, active recovery was given on Thursdays and complete rest on Sundays. The volume, intensity and load in the swimming sessions throughout the 21 weeks of training is shown in 0 Fig. 1a, b. Training intensity throughout this study was established by means of blood lactate measures, with $100 \%$ intensity corresponding to a swimming velocity at the anaerobic threshold for each individual. 
Table 1 Variation in heart rate and serum markers of muscle injury during the 21-week training season. The results are means (SD).

\begin{tabular}{llllll} 
Marker & \multicolumn{5}{c}{ Training Weeks } \\
& $\mathbf{1}$ & $\mathbf{6}$ & $\mathbf{1 1}$ & $\mathbf{1 6}$ & $\mathbf{2 1}$ \\
\hline HR $(\mathrm{bpm})$ & $66.03(2.07)$ & $60.83(1.16)^{\mathrm{a}}$ & $63.40(1.51)^{\mathrm{ab}}$ & $60.60(1.14)^{\mathrm{ac}}$ & $63.00(0.70)^{\mathrm{a}}$ \\
\hline \% & 100 & -7.87 & -3.98 & -4.58 & -3.03 \\
\hline LDH $(\mathrm{U} / \mathrm{L})$ & $175.00(7.67)$ & $212.00(17.74)^{\mathrm{a}}$ & $190.80(16.44)$ & $203.10(29.49)^{\mathrm{a}}$ & $179.00(14.60)$ \\
\hline$\%$ & 100 & +21.14 & +9.02 & +16.05 & +2.28 \\
\hline CK $(\mathrm{U} / \mathrm{L})$ & $161.10(25.57)$ & $335.30(140.10)^{\mathrm{a}}$ & $245.40(98.40)$ & $318.80(204.50)$ & $327.20(81.66)$ \\
\hline$\%$ & 100 & +108.13 & +52.32 & +97.88 & +103.10 \\
\hline Y- GT $(\mathrm{U} / \mathrm{L})$ & $17.30(3.61)$ & $16.29(4.07)$ & $14.78(3.80)$ & $15.76(3.54)$ & $20.10(3.02)$ \\
\hline$\%$ & 100 & -5.83 & -14.56 & -8.90 & +16.18 \\
\hline
\end{tabular}

a Significantly different from training week 1 at $<0.05$

${ }^{\mathrm{b}}$ Significantly different from training week 6 at $<0.05$

'Significantly different from training week 11 at $<0.05$

$\%$ Percentage of change

\section{Design}

Every 4 weeks during 5 months the participants attended the laboratory for the collection of saliva and blood. The participants refrained from intense exercise $72 \mathrm{~h}$ prior to the first collection of samples. However, the participants attended the laboratory during the subsequent 4 visits with no additional rest between training sessions. Resting HR was monitored employing a wireless signal transmission device (Polar RS300X, Polar Electro) and was recorded as the average of $2 \mathrm{~min}$ before the collection of samples.

\section{Collection of samples}

Whole saliva was collected with no exogenous stimulation. The saliva was allowed to pool in the mouth and then drooled into pre-weighted collection vials after $2 \mathrm{~min}$. The participants were asked to refrain from drinking, eating or tooth brushing during the hour prior to the collection of the samples. Immediately after collecting saliva, blood from the antecubital vein $( \pm 10 \mathrm{~mL})$ was withdrawn into EDTA-coated tubes. All collection procedures took place at $08: 00 \mathrm{~h}$ and were performed with the participants under fasting conditions. The participants had their blood routinely sampled for markers of muscle injury prior to this study and none of them reported the procedure as stressful. The analysis of the blood samples was performed immediately after the collection. After the collection of saliva, the samples were stored frozen at $-20^{\circ} \mathrm{C}$ until analysis.

\section{Serum markers}

The blood samples were centrifuged at $4^{\circ} \mathrm{C}$ for $5 \mathrm{~min}$ at $5000 \mathrm{rpm}$. The analysis of $\mathrm{LDH}, \mathrm{CK}$, and $\gamma-\mathrm{GT}$ was performed using the automatic chemistry analyzer Architect C-8000 (Abbott) using commercially available kits from the manufacturer and according to their protocol. The intra-assay coefficients of variation were below $10 \%$.

\section{Determination of sAA activity}

On the day of the analysis, the samples of saliva were thawed and centrifuged at $3000 \mathrm{rpm}$ for $15 \mathrm{~min}$ to remove mucins. For sAA analysis, $10 \mu \mathrm{L}$ of saliva were diluted $(1: 200)$ in MES buffer (MES $50 \mathrm{mM}, \mathrm{NaCl} 300 \mathrm{mM}, \mathrm{CaCl}_{2} 5 \mathrm{mM}$, KSCN $140 \mathrm{mM}$, pH 6.3) followed by the addition of $300 \mu \mathrm{L}$ of pre-heated $\left(37^{\circ} \mathrm{C}\right)$ substrate solution (2-chloro-p-nitrophenol, linked to maltotriose). The optical density was read at $405 \mathrm{~nm}$ at 1 -min intervals during $3 \mathrm{~min}$ at $37^{\circ} \mathrm{C}$ using a microplate reader (Molecular Devices, Menlo Park, CA). The enzyme activity was determined using the formula: [Absorbance difference per minute $\times$ total assay volume $(308 \mathrm{ml}) \times$ dilution factor (200)]/[millimolar absorptivity of 2-chloro-p-nitrophenol (12.9) $\times$ sample volume $(0.008 \mathrm{ml}) \times$ light path $(0.97)]$ [12]. The enzyme activity $(\mathrm{U} / \mathrm{mL})$ was then multiplied by flow rate $(\mathrm{mL} / \mathrm{min})$ to estimate the sAA secretion rate $(\mathrm{U} / \mathrm{min})$.

\section{Determination of the concentration of NO}

Nitric oxide was determined as nitrite formed using the Griess reaction. This assay is one of the most widely used techniques for the spectrophotometric measurement of nitrite in biological fluids [32]. $50 \mu \mathrm{L}$ of saliva were incubated with $50 \mu \mathrm{L}$ of Griess reagent ( $1 \%$ sulfanilamide in $2.5 \% \mathrm{H}_{3} \mathrm{PO}_{4}$ and $0.1 \% \mathrm{~N}$-(1-naphthyl)ethylenediamine dihydrochloride) at room temperature for $10 \mathrm{~min}$. The absorbance was measured at $570 \mathrm{~nm}$ using a microplate reader. The content of nitrite was calculated based on a standard curve constructed with $\mathrm{NaNO}_{2}$ at the concentrations of $400,200,100,50,25,12.5,6.25$ and $3.12 \mu \mathrm{M}$. The intra-assay coefficient of variation for duplicate samples was $3.8 \%$.

\section{Psychometric instruments}

The participants completed the POMS immediately before saliva collection. The participants were asked to state how they felt at the moment. The POMS is a 65-item questionnaire measuring tension, depression, anger, confusion, vigour and fatigue on a 5-point Likert scale. The internal consistency for the POMS (Cronbach's alpha coefficient) was reported at 0.96 [7].

\section{Statistical analysis}

The data were tested for normality using the Shapiro-Wilk test prior to the analyses. No transformations were necessary for any of the variables. All of the variables were compared by one-way analysis of variance (ANOVA) followed by the Tukey test for multiple comparisons. The relationships between the salivary markers and training outcomes were analysed using a 2-tailed Pearson correlation coefficient. The percentage of changes in salivary and serum markers was calculated by using the formula: percentage difference $=100 *$ (value - baseline $) /$ baseline . For all of the analyses, the significance level was $p<0.05$. The results shown are means (SD).

\section{Results}

$\nabla$

\section{Exercise-induced muscle damage}

- Table 1 shows the mean values of HR and serum markers throughout the training season. The resting HR decreased in 
Table 2 Mood disturbance scores of participants during the 21-week training season. The results are means (SD).

\begin{tabular}{|llllll} 
POMS Subscale Scores & $\mathbf{1}$ & $\mathbf{6}$ & $\mathbf{1 1}$ & $\mathbf{1 6}$ & $\mathbf{2 1}$ \\
tension - anxiety & $1.88(0.78)$ & $2.00(0.70)$ & $2.44(0.72)$ & $3.00(1.00)$ & $1.33(0.50)$ \\
\hline depression & $1.25(0.46)$ & $2.25(0.70)$ & $1.75(0.70)$ & $1.12(0.35)$ & $1.62(0.51)$ \\
anger - hostility & $0.50(0.71)$ & $0.60(0.51)$ & $0.10(0.31)$ & $0.2(0.42)$ & $0.90(0.61)$ \\
\hline vigour - activity & $12.13(0.83)$ & $13.88(1.12)$ & $11.63(1.88)$ & $10.38(1.30)$ & $10.63(1.18)$ \\
fatigue & $1.37(0.74)$ & $5.87(0.99)^{\mathrm{a}}$ & $2.75(0.70)^{\mathrm{b}}$ & $3.87(1.45)^{\mathrm{abc}}$ & $6.87(1.12)^{\mathrm{ac}}$ \\
\hline confusion - bewilderment & $1.75(0.70)$ & $1.62(0.91)$ & $1.75(0.46)$ & $0.62(0.74)^{\mathrm{ac}}$ & $0.75(0.52)^{\mathrm{ac}}$ \\
\hline total mood disturbance & $\mathbf{0 . 0 ( 0 . 0 )}$ & $\mathbf{0 . 6 2 ( 0 . 9 0 )}$ & $\mathbf{0 . 3 7}(\mathbf{0 . 7 4})$ & $\mathbf{0 . 3 7}(\mathbf{0 . 7 1 )}$ & $\mathbf{0 . 8 7}(\mathbf{0 . 9 9})$
\end{tabular}

asignificantly different from training week 1 at $p<0.05$

bSignificantly different from training week 6 at $p<0.05$

'Significantly different from training week 11 at $p<0.05$

response to training $[F(4,28)=15.70, \mathrm{p}<0.0001]$. The levels of $\operatorname{LDH}[F(4,33)=4.17, \mathrm{p}=0.007]$ and $\mathrm{CK}[F(4,30)=2.640, \mathrm{p}=0.048]$ differed significantly across the training season. However, no differences in the activity of $\gamma-G T[F(4,26)=1.82, \mathrm{p}=0.15]$ were observed in response to training.

\section{The response of NO and sAA to long-term training}

- Fig. 1c, $\mathbf{d}$ show the variation in salivary flow rate, SAA and NO in response to training. No significant changes in flow rate were observed $(F(4,29)=2.25, \mathrm{p}=0.086)$. However, sAA and NO showed a differential and opposite reactivity to training. Whereas the concentration of NO increased $[F(4,22)=5.05$, $\mathrm{p}=0.0048]$, there was a decline in $\mathrm{sAA}[F(4,26)=5.82, \mathrm{p}=0.0017]$ across the training season. The mean increase in NO was $239.9 \%$ (week 6), 181.5\% (week 11), 189.3\% (week 16), and $270.8 \%$ (week 21). On the other hand, the activity of sAA decreased by 30.9\% (week 6), 38.6 (week 11), 54.2\% (week 16) and 37.6\% (week 21) in response to training. Post hoc power calculations indicated that the statistical model used to relate training parameters with sAA activity and NO had a power of $40 \%$ and $48 \%$, respectively.

Correlation between salivary markers and training load Strong correlations between SAA and NO with the intensity of training were observed. Whereas sAA correlated negatively with the intensity $[r(11)=-0.78, \mathrm{p}<0.05]$, NO correlated positively [ $\mathrm{r}$ $(11)=0.92, p=0.025]$. Similar results were observed when sAA and NO were compared against the training load. A strong negative correlation was observed for sAA $[r(11)=-0.65, \mathrm{p}<0.05]$ and a strong positive correlation for NO $[r(11)=0.69, \mathrm{p}<0.05]$. No significant correlations between the salivary markers and the volume of training were observed.

\section{Mood disturbance}

The mood was not affected by training $[F(4,35)=1.45, \mathrm{p}=0.238]$. However, we observed higher scores of fatigue during the weeks with the highest training intensity (weeks 6,16 and 21) ( $\bullet$ Table 2).

\section{Discussion}

$\nabla$

The primary findings of this study are that both SAA and NO showed a proportional response to the oscillation of training intensity and load. In line with our expectations, independent of salivary flow, sAA decreased whereas NO increased across the training season. Salivary NO also behaved similarly to the serum measures of LDH and CK. These findings provide preliminary evidence to the potential feasibility of sAA and NO as markers of training intensity and load.

The rationale behind the use of SAA as a surrogate marker of sympathetic activity comes from evidence showing a similar response to exercise than blood noradrenaline [6]. Given the strong correlation between a catecholamine threshold with the dynamics of blood lactate during exercise, sAA also has been successfully applied to determine the anaerobic threshold in well-trained subjects [3]. Since the early study of Chatterton and colleagues [6], it has been demonstrated that in response to single bouts of exercise, the activity of sAA increases and remains elevated for up to $2 \mathrm{~h}$ thereafter $[1,19]$. A fact ascribed to changes in sympathetic activity.

No other study to our knowledge has previously assessed the response of sAA to long-term training in humans. However, the autonomic response after prolonged exercise is well documented. In essence, prolonged training has the ability to decrease sympathetic drive and increase parasympathetic activity with a concomitant reduced concentration of plasma catecholamines. This leads to a reduced peripheral resistance, decreased resting blood pressure and is also partially responsible for a lower resting HR [24]. Therefore, a decrease in SAA in response to long-term training could be expected, as it was observed in our study. Salivary glands such as the palate and the sublingual gland receive mainly parasympathetic stimulation and also secrete sAA [4]. Therefore, sAA should not be considered as an exclusive read-out of sympathetic activity. In fact, interpretation of data from traditional markers of sympathetic arousal such as HR also warrants some caution [2]. Although we are not able to distinguish any additive effects between the 2 branches of the ANS, it is well established that the rate of protein secretion into the saliva by sympathetic stimuli is superimposed upon parasympathetic stimulation when the glands are simultaneously innervated [31]. Psychological adverse stimuli have been known to alter the levels of sAA [23]. Since no variation in mood was reported across the training season, the variation in the sAA response to training appeared to be more strongly influenced by physiological than behavioural factors.

As with SAA, no other study appears to have investigated the salivary NO response to long-term training, so we are not able to compare our findings. Only few studies have determined the changes in salivary NO caused by acute exercise and they have yielded equivocal results with most studies $[25,27]$ but not all [11] reporting an increase in salivary NO after exercise. Interestingly, in our study salivary NO showed a proportional response to the training intensity and load. Nitric oxide is an important cellular messenger involved in the regulation of vascular tone 
amongst several other physiological functions. After acute and chronic exercise, there is an up-regulation of NOS and plasma nitrite levels. This has been attributed to shear stress caused by changes in haemodynamics and regeneration processes related to muscle injury $[14,21]$. In this respect, it is worth mentioning that we noted a very similar pattern in the levels of CK, LDH and salivary $\mathrm{NO}$ as well as a strong correlation between $\mathrm{NO}$ and LDH $[r(11)=0.71, \mathrm{p}<0.05]$.

Nitric oxide also has important roles as a signalling molecule in the nervous system. It is thought that NO regulates sympathovagal modulation by reducing the pre-synaptic release of noradrenaline [30]. Evidence now supports the notion that upregulation of NO is partially responsible for the reduction of sympathetic tone following training [22]. Due to the short halflife of NO, the quantification of NO metabolites has traditionally been used as indication of NO production. Nitrite is the main product of the oxidation of $\mathrm{NO}$ in plasma and previous research has demonstrated that the concentration of nitrite accurately reflects changes in NOS activity $[18,28]$. Moreover, the basal levels of plasma nitrite are reduced in subjects with endothelial dysfunction [17] whereas higher levels predict exercise capacity in trained men [28].

Taking the above into consideration, it would be expected to observe a decrease in SAA and a higher concentration of salivary NO in response to long-term training. However, even though salivary parameters are useful tools for understanding physiological processes, the oral cavity is particularly complex and variations in these parameters may not necessarily reflect variations in other systems of the body. Dietary nitrate, for instance, is concentrated in the salivary glands and is converted to nitrite in saliva by oral bacteria [8]. In addition, NO is involved in the regulation of salivary gland functioning [20] and we are not able to estimate the influence, if any, of NO metabolism in the salivary glands on the levels of salivary nitrite.

To control for possible confounding factors, the participants were monitored in the fasted state, the saliva was collected with no exogenous stimulation and the mood states were registered on each visit to the laboratory. Thus, neither dietary nitrate nor other factors known to stimulate sAA release different from exercise influenced our findings.

Previous research has shown than lower salivary flow rates are observed in healthy subjects due to dehydration during months with higher temperatures [15]. However, we did not find any difference in salivary flow rate and it is unlikely that such variation in SAA and salivary NO occurred as regular circannual rhythms. The serum levels of skeletal muscle enzymes are widely used as markers of muscle functional status. In our study, the dynamics of LDH and CK, but not $\gamma-G T$, were distinctly associated with the intensity of training. Although this finding is not particularly novel, the similarity of the variation in LDH and CK with salivary NO is noteworthy. As mentioned above, no statements can be made about their casual relationship at this point. Nevertheless, we believe the potential response of salivary NO to exercised-induced muscle injury is worth pursuing in future research, especially when previous studies have already demonstrated an up-regulation in NO in relation to muscle injury and recovery [9].

\section{Conclusions}

$\nabla$

Salivary alpha-amylase and NO showed proportional responses to the training intensity and load. Whereas the levels of sAA declined in response to training, salivary NO increased and behaved in a similar fashion to LDH and CK. This may be associated with an enhanced production in NO due to muscle injury and changes in haemodynamics. The oscillation of sAA and NO across the training season is consistent with previously reported variations in the regulation of sympathetic tone and concentrations of plasma nitrite. The changes in SAA, NO and training parameters highlight the use of these salivary constituents to monitor training programmes.

\section{Acknowledgements}

$\nabla$

We are grateful to the participants for their involvement. Thanks are also due to Mr. G. Degani and Mr. W. Pires for their support and consideration to include members of their team to conduct this work. This study was supported by grants from the funding agency Fapemig. MD and $\mathrm{OB}$ received graduate fellowships from CNPq and the international program PEC-PG/CNPq, respectively.

\section{References}

1 Allgrove JE, Gomes E, Hough J, Gleeson M. Effects of exercise intensity on salivary antimicrobial proteins and markers of stress in active men. J Sports Sci 2008; 26: 653-661

2 Berntson GG, Bigger JT Jr, Eckberg DL, Grossman P, Kaufmann PG, Malik $M$, Nagaraja HN, Porges SW, Saul JP, Stone PH, van der Molen MW. Heart rate variability: origins, methods, and interpretive caveats. Psychophysiology 1997; 34: 623-648

3 Bocanegra OL, Diaz MM, Teixeira RR, Soares SS, Espindola FS. Determination of the lactate threshold by means of salivary biomarkers: chromogranin A as novel marker of exercise intensity. Eur J Appl Physiol [Epub ahead of print]. doi:10.1007/s00421-011-2294-4

4 Bosch JA, Veerman ECI, de Geus EJ, Proctor GB. $\alpha$-Amylase as a reliable and convenient measure of sympathetic activity: don't start salivating just yet! Psychoneuroendocrinology 2011; 36: 449-453

5 Brancaccio P, Lippi G, Maffulli N. Biochemical markers of muscular damage. Clin Chem Lab Med 2010; 48: 757-767

6 Chatterton RT Jr, Vogelsong KM, Lu YC, Ellman AB, Hudgens GA. Salivary alpha-amylase as a measure of endogenous adrenergic activity. Clin Physiol 1996; 16: 433-448

7 Droppleman M, Douglas M, Lorr M, Leo F. Psychiatric Rating Scales: Profile of Mood States (POMS) No. 8 in a Series 1971; Hoffmann-La Roche Inc.

8 Duncan C, Dougall H, Johnston P, Green S, Brogan R, Leifert C, Smith $L$, Golden M, Benjamin N. Chemical generation of nitric oxide in the mouth from the enterosalivary circulation of dietary nitrate. Nat Med 1995; 1: 546-551

9 Filippin LI, Moreira AJ, Marroni NP, Xavier RM. Nitric oxide and repair of skeletal muscle injury. Nitric Oxide 2009; 21: 157-163

10 Goldsmith RL, Bloomfield DM, Rosenwinkel ET. Exercise and autonomic function. Coron Artery Dis 2000; 11: 129-135

11 González D, Marquina $R$, Rondón $N$, Rodriguez-Malaver AJ, Reyes $R$. Effects of aerobic exercise on uric acid, total antioxidant activity, oxidative stress, and nitric oxide in human saliva. Res Sports Med 2008; 16: 128-137

12 Granger DA, Kivlighan KT, el-Sheikh M, Gordis EB, Stroud LR. Salivary alpha-amylase in biobehavioral research: recent developments and applications. Ann N Y Acad Sci 2007; 1098: 122-144

13 Harriss DJ, Atkinson G. Update - ethical standards in sport and exercise science research. Int J Sports Med 2011; 32: 819-821

14 Huonker $M$, Halle $M$, Keul J. Structural and functional adaptations of the cardiovascular system by training. Int J Sports Med 1996; 17 (Suppl 3): S164-S172

15 Kariyawasam AP, Dawes C. A circannual rhythm in unstimulated salivary flow rate when the ambient temperature varies by only about 2 degrees C. Arch Oral Biol 2005; 50: 919-922

16 Kenttä G, Hassmén P. Overtraining and recovery. A conceptual model. Sports Med 1998; 26: 1-16

17 Kleinbongard P, Dejam A, Lauer T, Jax T, Kerber S, Gharini P, Balzer J, Zotz RB, Scharf RE, Willers R, Schechter AN, Feelisch M, Kelm M. Plasma nitrite concentrations reflect the degree of endothelial dysfunction in humans. Free Radic Biol Med 2006; 40: 295-302 
18 Lauer T, Preik M, Rassaf T, Strauer BE, Deussen A, Feelisch M, Kelm M. Plasma nitrite rather than nitrate reflects regional endothelial nitric oxide synthase activity but lacks intrinsic vasodilator action. Proc Natl Acad Sci USA 2001; 98: 12814-12819

19 Li T-L, Gleeson M. The effect of single and repeated bouts of prolonged cycling and circadian variation on saliva flow rate, immunoglobulin A and alpha-amylase responses. J Sports Sci 2004; 22: 1015-1024

20 Looms D, Tritsaris K, Pedersen AM, Nauntofte B, Dissing S. Nitric oxide signalling in salivary glands. J Oral Pathol Med 2002; 31: 569-584

21 Maiorana A, O'Driscoll G, Taylor R, Green D. Exercise and the nitric oxide vasodilator system. Sports Med 2003; 33: 1013-1035

22 Mohan RM, Choate JK, Golding S, Herring N, Casadei B, Paterson DJ. Peripheral pre-synaptic pathway reduces the heart rate response to sympathetic activation following exercise training: role of NO. Cardiovasc Res 2000; 47: 90-98

23 Nater UM, Rohleder N. Salivary alpha-amylase as a non-invasive biomarker for the sympathetic nervous system: current state of research. Psychoneuroendocrinology 2009; 34: 486-496

24 O'Sullivan SE, Bell C. Training reduces autonomic cardiovascular responses to both exercise-dependent and -independent stimuli in humans. Auton Neurosci 2001; 91: 76-84

25 Panossian AG, Oganessian AS, Ambartsumian M, Gabrielian ES, Wagner $H$, Wikman $G$. Effects of heavy physical exercise and adaptogens on nitric oxide content in human saliva. Phytomedicine 1999; 6: 17-26

26 Proctor GB, Carpenter GH. Regulation of salivary gland function by autonomic nerves. Auton Neurosci 2007; 133: 3-18
27 Rahman ZA, Abdullah N, Singh R, Sosroseno W. Effect of acute exercise on the levels of salivary cortisol, tumor necrosis factor-alpha and nitric oxide. J Oral Sci 2010; 52: 133-136

28 Rassaf T, Lauer T, Heiss C, Balzer J, Mangold S, Leyendecker T, Rottler J, Drexhage C, Meyer C, Kelm M. Nitric oxide synthase-derived plasma nitrite predicts exercise capacity. Br J Sports Med 2007; 41: 669-673

29 Scannapieco FA, Torres G, Levine MJ. Salivary alpha-amylase: role in dental plaque and caries formation. Crit Rev Oral Biol Med 1993; 4: 301-307

30 Schwarz P, Diem R, Dun NJ, Förstermann U. Endogenous and exogenous nitric oxide inhibits norepinephrine release from rat heart sympathetic nerves. Circ Res 1995; 77: 841-848

31 Smith DJ. A framework for understanding the training process leading to elite performance. Sports Med 2003; 33: 1103-1126

32 Tsikas $D$. Analysis of nitrite and nitrate in biological fluids by assays based on the Griess reaction: appraisal of the Griess reaction in the L-arginine/nitric oxide area of research. J Chromatogr B Analyt Technol Biomed Life Sci 2007; 851: 51-70

33 Urhausen A, Gabriel H, Kindermann W. Blood hormones as markers of training stress and overtraining. Sports Med 1995; 20: 251-276

34 Urhausen A, Kindermann W. Diagnosis of overtraining: what tools do we have? Sports Med 2002; 32: 95-102 